\title{
THE EFFECT OF ENAMEL PRE-TREATMENT WITH SILVER DIAMINE FLUORIDE VERSUS NANO SILVER FLUORIDE ON THE MICROLEAKAGE OF FISSURE SEALANT: IN VITRO STUDY
}

\author{
Magda El-Tekeya* and Laila M. El-Habashy**
}

\begin{abstract}
Many studies in literature showed the effectiveness of silver diamine fluoride (SDF) and Nano silver fluoride (NSF) as preventive agents. However, there is little evidence regarding the retention properties of fissure sealant (FS) when applied on a surface previously treated with these preventive agents.
\end{abstract}

Objective: To evaluate and compare the effect of fissure pre-treatment with SDF versus NSF on the microleakage of FS in permanent teeth.

Methods: This experimental in vitro study was performed on 45 extracted human premolars, teeth were randomly assigned into three groups according to the type of fissure treatment: Group I: Control group (sealant only), Group II: SDF group (pre-treatment before sealant), and Group III: NSF group (pre-treatment before sealant). Teeth were thermos-cycled and then immersed in $2 \%$ methylene blue solution for 24 hours, teeth were then sectioned mesio-distally in the middle of the fissure, and examined under the stereomicroscope for dye penetration.

Results: There was no statistical significant difference in the microleakage scores between the three groups $(p=0.247)$. Conclusion: Pretreatment of the pits and fissure with SDF or NSF prior to sealant application did not affect the micro-leakage of the FS.

\section{INTRODUCTION}

Pit and fissure caries is the most prevalent caries in primary and permanent dentition accounting for 80 to 90 percent of the total caries incidence, this is due to the morphology of the pits and fis- sures that form an area of food and plaque entrapment which is difficult to clean by regular tooth brushing. ${ }^{(1)}$ Cariogenic bacteria ferments carbohydrates producing acid that causes demineralization of tooth surfaces. ${ }^{(2)}$ Caries is a dynamic process that occurs when periods of demineralization exceed

\footnotetext{
* Professor, Pediatric Dentistry, and Dental Public Health Department, Faculty of Dentistry, Alexandria University, Alexandria, Egypt.

** Assistant Professor, Pediatric Dentistry and Dental Public Health Department, Faculty of Dentistry, Alexandria University, Alexandria, Egypt
} 
the periods of re-mineralization resulting in a white spot lesion, followed by cavitation. . $^{(3,4)}$

Fissure sealant (FS) was developed as a preventive caries measure by forming a physical barrier that prevent food and plaque accumulation. ${ }^{(5,6)}$ However, the success of FS depends on its ability to provide a tight sealing to the pits and fissures, improper sealing will lead to marginal leakage with resultant caries progression..$^{(7,8)}$

Silver diamine fluoride in a concentration of $38 \%$ (44,800 ppm fluoride) was used 80 years ago in Japan to arrest dental caries, ${ }^{(9)}$ and since 2014 it was used in the United States. ${ }^{(10)}$ The material is formed from three components: silver (Ag), diammonia (2NH3) and fluoride (Fl). It offers the synergistic antimicrobial effect of both silver and fluoride inhibiting the cariogenic bacteria, plus offering the advantage of fluoride as re-mineralizing agent to the de-mineralized tooth surface and converting hydroxyl appetite crystal to fluoro- appetite making the surface less liable to demineralization. The ammonia was added to reduce oxidation potential and thus producing a more stable compound with longer shelf life. ${ }^{(9-11)}$ Many studies have proven that SDF solution is a simple, inexpensive, and effective method in preventing pit and fissure caries compared with other materials, ${ }^{(12-14)}$ it also follows the principle of minimally invasive dentistry. (15) Furthermore, a recent in-vitro study concluded that a tooth surface treated with SDF before the application of FS increased the bonding strength and decreased microleakage. ${ }^{(16)}$ However, the main disadvantage of SDF is its black staining to the carious tissue due to the oxidation reaction of silver ions. ${ }^{(17)}$

The development of Nanotechnology has added a great benefit in the field of dental material. ${ }^{(18)}$ Nanoparticles were detected in the 1980s, by the electron microscope with special effects. A nanoparticle is a body having a dimension of the order of 100 nanometers equivalent to about one thousand atoms. The nanoparticles have interesting properties that are entirely dependent, because at the scale of nanoparticles, the physical attributes of these properties are different from the original material. ${ }^{(19)}$

Nano-silver fluoride (NSF) was recently introduced as a new experimental preparation, it consists of Nano Silver particles, Chitosan and Fluoride. Chitosan is a substance found in crabs' skin and is used in medical field for its antimicrobial and anti-inflammatory action, and as stabilizing agent. NSF is a yellow solution that proved to be stable for 3 years it was developed to overcome the staining problems of SDF as the material doesn't undergo oxidation, and has proved to be ecofriendly. ${ }^{(20,21)}$ It combines the antimicrobial effect of chitosan and Nano silver ions, plus the preventive action of its fluoride content. ${ }^{(22)}$ In 2012, Santos et al found that the anti-cariogenic effect of NSF was similar to that of SDF when applied once a year. ${ }^{(23,24)}$ In 2014, Targino showed that NSF preparation was efficacious against $\mathrm{S}$. Mutans in much smaller doses than SDF. ${ }^{(20)}$

Most studies in literature showed the effectiveness of both SDF and NSF as preventive agents, however, there is little evidence on their effect when applied as pre-treatment prior to sealant application, and their effect on the retention and the sealing property of FS. Since sealants with higher adhesion presents less microleakage scores, and thus better clinical performance, therefore, the aim of this in vitro study was to evaluate and compare the effect of fissure pre-treatment with SDF versus NSF on the microleakage of FS in permanent teeth.

The null hypothesis of the present study was there will be no difference between SDF and NSF when used as fissure pretreatment on the microleakage of FS.

\section{METHODS}

This experimental in-vitro study was conducted at Pediatric Dentistry and Dental Public Health, Dental Biomaterials Departments, Faculty of Dentistry and Faculty of Pharmacy, Alexandria 
University, Egypt.

Ethics approval was taken from the Ethical research Committee, Faculty of Dentistry, Alexandria University (IORG 0008839).

Sample size calculation: Sample size was estimated based on the following assumptions: alpha error $=5 \%$, study power $=80 \%$. According to PérezHernández et al, ${ }^{(16)}$ microleakage did not occur in 27 (50.94\%) specimens when silver diamine fluoride pretreatment was used and in $6(10.9 \%)$ specimens when no pretreatment was used. Silver nanofluoride pretreatment is assumed to have a similar effect on adhesion of composite resin. ${ }^{(25)}$ Based on comparison of proportions, with an effect size $=1.09$, sample size was calculated to be 14 per group which was increased to 15 to make up for laboratory processing errors. The total sample size $=$ number of groups $\times$ number per group $=3 \times 15=45$. Sample size was calculated using GPower version 3.1.9.4. ${ }^{(26)}$

\section{Sample selection}

Sixty human extracted premolars (for orthodontic treatment purpose) were collected from from the Department of Oral Surgery out-patient clinics. Teeth were hand scaled, cleaned using pumice with rubber cup on low speed hand piece then washed with water and stored in normal saline. The teeth were then carefully examined using magnifying lens; 45 exfoliated premolars were selected without caries or with caries grade one with no developmental anomalies. Exclusion criteria included: teeth with hypoplasia, cracks, or malformations.

Nano Silver Fluoride preparation: the material was prepared at faculty of Pharmacy, Alexandria University. NSF aqueous solution was prepared according to Wei et al ${ }^{(27)}$ by the chemical reduction of silver nitrate $(1 \mathrm{~mL}, 0.11 \mathrm{M})$ with sodium borohydride $(0.3 \mathrm{~mL}, 0.8 \mathrm{M})$ and chitosan biopolymer $(28.7 \mathrm{~mL}, 2.5 \mathrm{mg} / \mathrm{mL})$ as a stabilizing agent. Sodium fluoride $(10,147 \mathrm{ppm}$ of fluorine) was then added at the end of the experiment. The net results of this reaction yielded Nano silver fluoride.
Grouping: The teeth were randomly assigned (using computer generated random numbers) into three main groups of 15 teeth each according to the type of treatment:

Group I: Control group: Sealant (Clinpro ${ }^{\mathrm{TM}} 3 \mathrm{M}$ ESPE) was applied without fissure pre-treatment.

Group II: SDF (Advantage Arrest SDF 38\%, Elevate Oral Care, LLC, West Palm Beach, Florida, USA) was used as pre-treatment agent before sealant application, the material $\left(\mathrm{Ag}(\mathrm{NH} 3)_{2} \mathrm{~F}\right)$ is a colourless solution formed of 24.4-28.8\% (w/v) silver and $5.0-5.9 \%$ fluoride $(44800 \mathrm{ppm})$ with $\mathrm{PH}=10$.

Group III: NSF was used as pre-treatment agent before sealant application.

Procedure: All 45 premolar's roots were cut off $2 \mathrm{~mm}$ below the cemento-enamel junction with water-cooled low speed diamond saw. Teeth were mounted in acrylic blocks with the occlusal surfaces perpendicular to the long axis of the blocks. In the Control group $(n=15)$ all molars were sealed according to manufacturer's recommendation: the etchant (Scotchbond ${ }^{\mathrm{TM}}$ 35\% 3M ESPE) was applied along the enamel occlusal pits and fissure for $30 \mathrm{~s}$, rinsed with water spray, and then the enamel was thoroughly dried with air spray for five seconds. Sealant was then applied slowly using syringe needle into the pits and fissures and was light cured for 20 s. In the SDF Group ( $n=15)$ : A drop of SDF was applied on the central groove of the occlusal surfaces of the teeth using a micro-brush, and kept for 4 minutes. It was then washed with a wet cotton pellet and dried with air syringe. ${ }^{(16)}$ This was followed by etching rinsing and sealant application. In the NSF group $(\boldsymbol{n}=15)$ : A drop of NSF solution was applied to the central groove of each tooth using a micro brush. The solution was left in contact with tooth surface for 2 minutes then rinsed with water and air dried. ${ }^{(23)}$ This was followed by etching rinsing and sealant application. 
Microleakage test: All teeth were thermoscycled for 500 cycles from $5^{\circ} \mathrm{C}$ to $55^{\circ} \mathrm{C}$ with $30 \mathrm{~s}$ dwell time, $20 \mathrm{~s}$ transfer time, corresponding to 6 months' clinical use. ${ }^{(28)}$ Two layers of nail varnish was then applied over the teeth leaving only one $\mathrm{mm}$ free around the occlusal sealant border. ${ }^{(29)}$ The occlusal surface of each tooth was then immersed in $1 \%$ methylene blue dye solution $(\mathrm{pH}=7)$ at $37^{\circ} \mathrm{C}$ for 24 hours and stored in incubator, this permitted the dye penetration into the existing voids between the tooth substance and the restorative material. ${ }^{(30)}$ The teeth were then washed under tap water and were sectioned mesio-distally in the center of the fissure with low speed water-cooled diamond saw giving 30 specimens in each group. Each section was examined using Stereomicroscope (SZ1145TR, Olympus, Japan) at 10X magnification. An image of every section was captured using a video camera connected to the computer. Each section was examined for dye penetration. The micro-leakage score was given by a single examiner who was blinded to the treatment and who was trained according to intra-rater agreement ${ }^{(31)}$ to the following scoring system ${ }^{(32)}$ :

$0=$ no evidence of dye penetration; $1=$ dye penetration of less than $1 / 3$ from the margin of restoration; $2=$ dye penetration of more than $1 / 3$ and less than $2 / 3$ from the margin of restoration; $3=$ dye penetration of more than $2 / 3$ from the margin of restoration (Figure 1).

\section{Statistical Analysis}

Data were collected and analyzed using the SPSS version 21. Pearson's chi-squared test and $\mathrm{Z}$ test for comparison of column proportion with adjusted $\mathrm{p}$ value using Bonerroni method were used for comparison of microleakage scores among the 3 groups. Monte Carlo correction for $\mathrm{p}$ value of Chisquared test was used. 95\% level was chosen as a level of significance.

\section{RESULTS}

The distribution of dye penetration among the study and control groups was shown in Table 1:

Score 0: There was no dye penetration in $46.66 \%$ of Group I (sealant without fissure pretreatment), $66.66 \%$ of grouop II (sealant with SDF pretreatment), and $73.33 \%$ of group III (sealant with NSF pretreatment).

Score 1: There was dye penetration less than $1 / 3$ from the margin of restoration in $26.66 \%$ of group I, $13.33 \%$ of group II, and $20 \%$ of group III

Score 2: There was dye penetration more than $1 / 3$ and less than $2 / 3$ from the margin of restoration in $13.33 \%$ of group I, $13.33 \%$ of group II, and $6.66 \%$ of group III.

Score 3: There was dye penetration more than $2 / 3$ from the margin of restoration in $13.33 \%$ of group I, $6.66 \%$ of group II, and None in group III.

TABLE (1) Comparison of microleakage scores among the three studied groups

\begin{tabular}{|l|c|c|c|}
\hline $\begin{array}{l}\text { Microleakage } \\
\text { score }\end{array}$ & $\begin{array}{c}\text { Sealant } \\
\text { Group 1) } \\
(\mathrm{n}=30)\end{array}$ & $\begin{array}{c}\text { SDF } \\
\text { Pretreatment } \\
(\text { Group 2) } \\
(\mathrm{n}=30)\end{array}$ & $\begin{array}{c}\text { NSF } \\
\text { Pretreatment } \\
(\text { Group 3) } \\
(\mathrm{n}=30)\end{array}$ \\
\hline Score 0 & $14^{\mathrm{a}}(44.67 \%)$ & $20^{\mathrm{a}}(66.67 \%)$ & $22^{\mathrm{a}}(73.33 \%)$ \\
\hline Score 1 & $8^{\mathrm{a}}(26.67 \%)$ & $4^{\mathrm{a}}(13.33 \%)$ & $6^{\mathrm{a}}(20.00 \%)$ \\
\hline Score 2 & $4^{\mathrm{a}}(13.33 \%)$ & $4^{\mathrm{a}}(13.33 \%)$ & $2^{\mathrm{a}}(6.67 \%)$ \\
\hline Score 3 & $4^{\mathrm{a}}(13.33 \%)$ & $2^{\mathrm{a}}(6.67 \%)$ & $0^{\mathrm{a}}(0.00 \%)$ \\
\hline $\begin{array}{l}\text { Test of } \\
\text { significance }\end{array}$ & \multicolumn{3}{|c|}{$\mathrm{c}^{2}{ }_{(\mathrm{df}=6)}=7.990$} \\
$p_{(\mathrm{MC})}=0.247 \mathrm{NS}$ \\
\hline
\end{tabular}

Subscript (a) denotes a subset of Group categories whose column proportions do not differ significantly from each other at the .05 level using $Z$ test for comparison of column proportions with adjusted $p$ values using Bonferroni method.

$c^{2}$ : Pearson's Chi-Squared test

MC: Monte Carlo correction

NS: Statistically not significant 
There was no statistically significant difference in scores among the three studied groups $\left(\mathrm{c}^{2}=7.990\right.$, $p=0.247)$. Using $\mathrm{Z}$ test for column proportion, each score level, either $0,1,2$ or 3 showed no statistical difference among the three groups.

An observation that was found in the present study was of a clear white zone seen at the base of the fissure in all specimens of the NSF group, and in 13 specimens out of 15 in the SDF group. This zone was not detected in the control group when the sealant was placed directly to the fissure without SDF or NSF pre-treatment (figure 2)

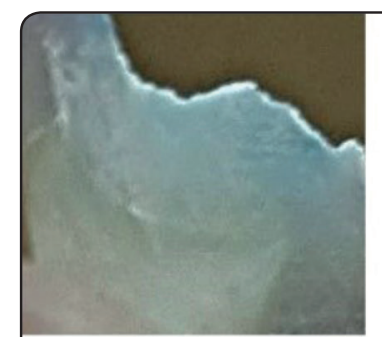

Score 0

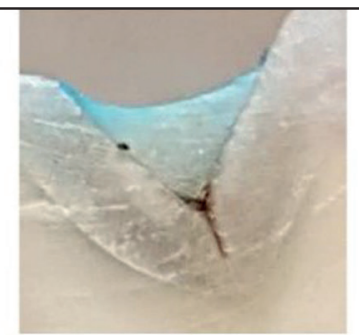

Score 1

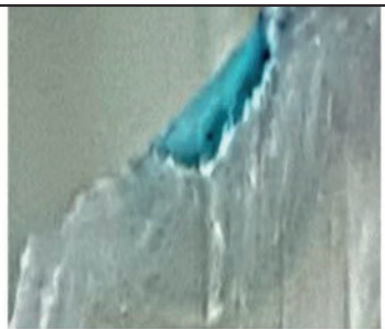

Score 2

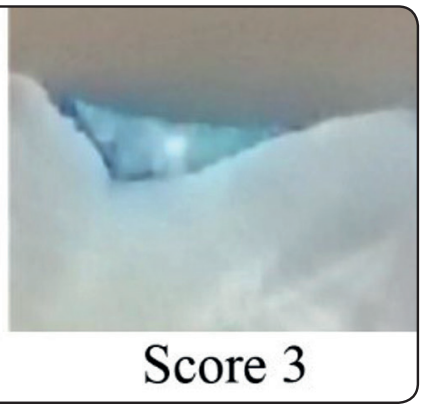

Score 3

Fig. (1) Dye penetration scores: $0=$ no evidence of dye penetration; $1=$ dye penetration of less than $1 / 3$ from the margin of restoration; $2=$ dye penetration of more than $1 / 3$ and less than $2 / 3$ from the margin of restoration; $3=$ dye penetration of more than $2 / 3$ from the margin of restoration.

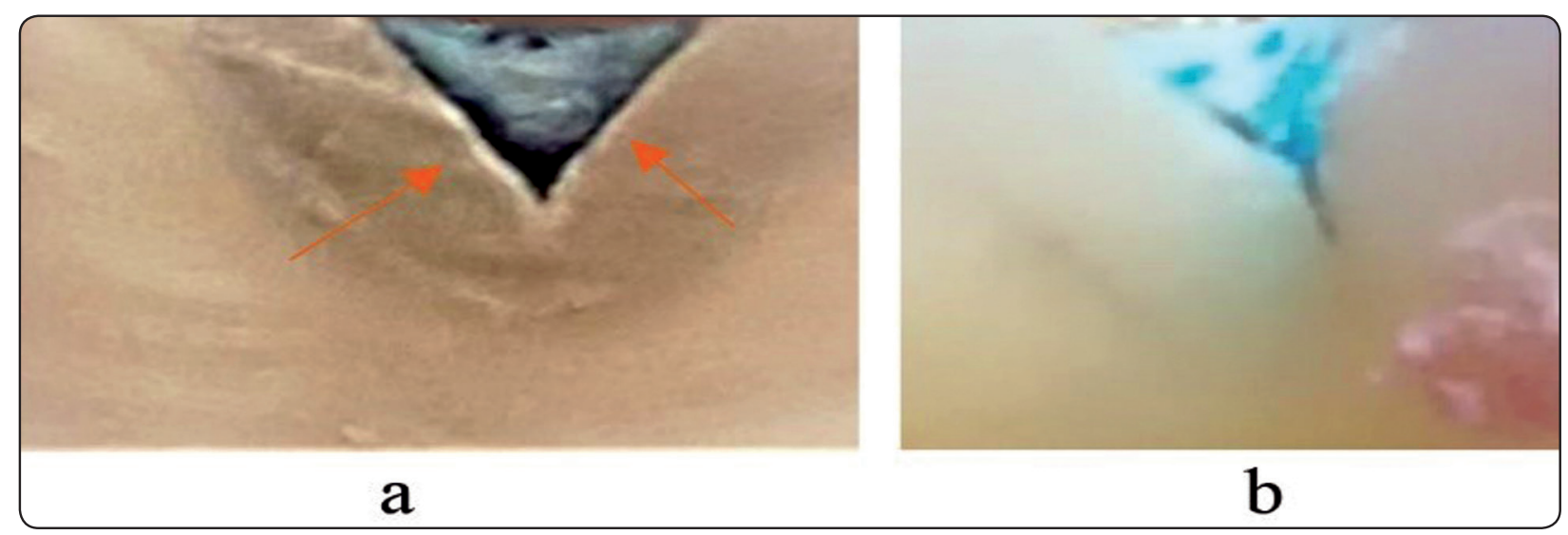

Fig. (2) (a) illustrating the white zone which was observed at the base of the fissure in the test groups, and which was absent in the control group (b)

\section{DISCUSSION}

Prevention is the cornerstone of modern dentistry; minimal intervention has become the standard of care in dental practice. SDF has gained popularity in the past few years as it offers the synergistic antimicrobial effect of both silver and fluoride inhibiting the cariogenic bacteria, plus offering the advantage of fluoride as re-mineralizing agent. ${ }^{(9,10)}$ However, SDF has its limitations as it produces black discol- orations which is not convenient by many patients or dentists.

Based on the nanotechnology of dental material, NSF was recently introduced, the material has proved to offer the same advantages of SDF, moreover, because of its nano particle structure it has proved to be more ecofriendly, more stable and has overcome the discoloration effect of SDF by resisting the oxidation process. ${ }^{(19,23)}$ 
The idea of pretreating the fissures with SDF or NSF before sealant applications would combine the antimicrobial/re-meneralizing action of these agents and the physical sealing property of FS, this would be of great benefit in caries prevention especially in high risk patients. Scarce studies are available on the effect of SDF or NSF when used as fissure pretreatment and their effect on the adhesion and sealing property of FS. Therefore, this study aimed to compare the effect of SDF versus NSF when used as pre-treatment on the microleakage of FS to tooth enamel.

Dye penetration test was used in this study as it is well known that sealants with higher adhesion presents less microleakage scores, and thus better clinical performance. Both SDF and NSF were used to give a more comprehensive outcome on these two preventive agents and if the evolution of the Nano-particles of the material would yield better performance.

In the present study, the micro-leakage test was performed after all teeth were thermos-cycled for 500 cycles from $5{ }^{\circ} \mathrm{C}$ to $55^{\circ} \mathrm{C}$ with $30 \mathrm{~s}$ dwell time, $20 \mathrm{~s}$ transfer time, as this would correspond to six months' clinical use and therefore would give more practical results on the intraoral performance of the restoration. ${ }^{(29)}$

The result of this study showed that fissure pretreatment with either SDF or NSF presented better results with no dye penetration than FS without pre-treatment: $66.66 \%, 73.33 \% \%$ and $46.66 \%$ respectively, however, this difference didn't reach the level of significance. This finding indicated that the sealing ability of FS was not affected when enamel was pretreated with either SDF or NSF.

The results of fissure pretreatment with SDF of the present study were in accordance with Uzuel et al. $2013^{(33)}$ who concluded that SDF did not significantly change the micro-leakage of composite resin when it was applied at the base of posterior class $\mathrm{V}$ cavities prior to composite resin filling. On the other hand, our results were inconsistent with Pérez, et al. ${ }^{(16)}$ who found a significant greater microleakage (reaching almost the double) when FS was applied without SDF pre-treatment (81.66\%), compared to the sealant with SDF pre-treatment (47\%), while in the present study the microleakage was in $53.34 \%$ in sealant without SDF pre-treatment, compared to 33.34 in sealant with SDF pre-treatment with no significant difference. The difference in the outcomes between the two studies might be due to the different techniques in SDF applications. In their study the fissure was treated twice by SDF with one-week interval between the two applications, while in the present study SDF was placed once for four 4 minutes then sealant was applied.

Our finding that fissure pre-treatment with NSF didn't affect micro-leakage scores of the FS was supported by Morales et al, ${ }^{(34)}$ and Khairy et al. ${ }^{(35)}$ who concluded that that there was no significant difference in the marginal seal or micro-leakage between the silver nanoparticle-containing sealant and the conventional sealant when applied in primary molars or permanent premolars respectively.

Although there was no statistical significant difference in the microleakage scores between the two test groups of this study, yet the pre-treatment with NSF showed better results than SDF, this finding was supportive to the fundamentals of nanotechnology that the nano particles of a material provide better cohesion property with less voids. ${ }^{(19)}$

An interesting finding in this study was the observation of a white zone at the base of the fissure in both test groups which was not detected in the control group when the sealant was placed directly to the fissure without SDF or NSF pre-treatment. The explanation of this white zone might be attributed to the precipitation of silver particles from SDF, and nano-silver particles from NSF on the enamel surface forming a well-defined zone that prevented any dye penetration, as if an insulating zone at the base of the fissure was formed. This finding was supported by Suzuki et al, ${ }^{(36)}$ who evaluated the effect of SDF on enamel surface and found that after the application of SDF large amounts of silver (analyzed by the electron probe micro-analyzer) were precipitated on the enamel surface, and some silver ions diffuse into 
the enamel surface approximately $20 \mu \mathrm{m}$ of depth. This finding suggests that fissures pretreated with SDF or NSF before sealant placement will enhance the prevention of microleakage and thus reduces the possibility of caries under sealant when clinically applied. However, further studies are still needed for more explanation of the precipitation of silver particles from SDF or NSF on enamel surface and its effect on the penetration of resin tags into the etched enamel surface which is a major factor that determines the resin retention to the tooth surface.

Since adhesion of any restoration is mainly determined by shear bond strength and microleakage, therefore it has to be noted that from the limitation of the of the present study was the absence of the shear bond strength test as it would have definitely high-lighted the effect of fissure pretreatment with SDF or Nano silver fluoride on Sealant adhesion in a more affirmative way.

The null-hypothesis of this study was accepted as there was no significant difference between fisuure pretreatment with SDf or NSF on the microleakage of FS.

\section{CONCLUSION}

Based on the results of the present study it was concluded that:

Pretreatment of the pits and fissure with silver diamine fluoride or nano-silver fluoride prior to sealant application did not affect the microleakage of the Fissure sealant.

\section{Recommendation}

More laboratory studies are need to determine the effect of fissure pretreatment with SDF or Nano silver fluoride on the shear bond strength of FS.

More histological studies are also needed to examine the sealant/enamel interface after pretreatment with these preventive agents, to give more explanatory results on the depth of enamel resin tags which are considered the cornerstone for the retention of all resin material to the tooth surface.

\section{REFERENCES}

1. Axelsson P, Swenden K. Preventive Materials, Methods, and Programs. $1^{\text {st }}$ ed. Chicago: Quintessence Pub.; 2004.

2. Nakornchai S, Atsawasuwan P, Kitamura E, Surarit R, Yamauchi M. Partial biochemical characterisation of collagen in carious dentin of human primary teeth. Arch Oral Biol 2004; 49(4): 267-73.

3. Núñez DP, García Bacallao L. Bioquímica de la caries dental. . J Revista Habanera de Ciencias Médicas 2010; 9: $156-66$.

4. Cury JA, Tenuta LM. Enamel remineralization: controlling the caries disease or treating early caries lesions? Braz Oral Res 2009; 23 Suppl 1:23-30.

5. Beauchamp J, Caufield PW, Crall JJ, Donly K, Feigal R, Gooch B, et al. Evidence-based clinical recommendations for the use of pit-and-fissure sealants: a report of the American Dental Association Council on Scientific Affairs. J Am Dent Assoc 2008; 139(3): 257-68.

6. Ahovuo-Saloranta A, Forss H, Walsh T, Hiiri A, Nordblad A, Mäkelä M, et al. Sealants for preventing dental decay in the permanent teeth. Cochrane Database Syst Rev 2013; (3): Cd001830.

7. Li F, Li F, Wu D, Ma S, Gao J, Li Y, et al. The effect of an antibacterial monomer on the antibacterial activity and mechanical properties of a pit-and-fissure sealant. J Am Dent Assoc 2011; 142(2): 184-93.

8. Irish Oral Health Services Guideline Initiative. Pit and fissure sealants evidence-based guidance on the use of sealants for the prevention and management of pit and fissure caries. Retrieved from: https://wwwlenusie/bitstream/ handle/10147/113084/FSGuidelineOnlineFinalV2pdf;jses sionid=9A907DB6DFE0205FE2FE3AF8A5D2C015?seq uence $=12010$.

9. $\mathrm{Chu} \mathrm{CH}$, Lo EC. Promoting caries arrest in children with silver diamine fluoride: a review. Oral Health Prev Dent 2008; 6(4): 315-21.

10. Yee R, Holmgren C, Mulder J, Lama D, Walker D, van Palenstein Helderman W. Efficacy of silver diamine fluoride for Arresting Caries Treatment. J Dent Res 2009; 88(7): 644-7.

11. Craig GG, Knight GM, McIntyre JM. Clinical evaluation of diamine silver fluoride/potassium iodide as a dentine desensitizing agent. A pilot study. Aust Dent J 2012; 57(3): 308-11.

12. Liu BY, Lo EC, Chu CH, Lin HC. Randomized trial on fluorides and sealants for fissure caries prevention. J Dent Res 2012; 91(8): 753-8. 
13. Zhao IS, Gao SS, Hiraishi N, Burrow MF, Duangthip D, Mei ML, et al. Mechanisms of silver diamine fluoride on arresting caries: a literature review. Int Dent J 2018; 68(2): 67-76.

14. Mei ML, Lo EC, Chu CH. Clinical Use of Silver Diamine Fluoride in Dental Treatment. Compend Contin Educ Dent 2016; 37(2): 93-8; quiz100.

15. Ericson D, Kidd E, McComb D, Mjör I, Noack MJ. Minimally Invasive Dentistry--concepts and techniques in cariology. Oral Health Prev Dent 2003; 1(1): 59-72.

16. Pérez-Hernández J, Aguilar-Díaz FC, Venegas-Lancón RD, Gayosso C, Villanueva-Vilchis MC, de la Fuente-Hernández J. Effect of silver diamine fluoride on adhesion and microleakage of a pit and fissure sealant to tooth enamel: in vitro trial. Eur Arch Paediatr Dent 2018; 19(6): 411-6.

17. Rosenblatt A, Stamford TC, Niederman R. Silver diamine fluoride: a caries "silver-fluoride bullet". J Dent Res 2009; 88(2): 116-25.

18. Allaker RP. The use of nanoparticles to control oral biofilm formation. J Dent Res 2010; 89(11): 1175-86.

19. Valiev R. Materials science: nanomaterial advantage. Nature 2002; 419(6910): 887, 9 .

20. Targino AG, Flores MA, dos Santos Junior VE, de Godoy Bené Bezerra F, de Luna Freire H, Galembeck A, et al. An innovative approach to treating dental decay in children. A new anti-caries agent. J Mater Sci Mater Med 2014; 25(8): 2041-7.

21. Chu CH, Lo EC, Lin HC. Effectiveness of silver diamine fluoride and sodium fluoride varnish in arresting dentin caries in Chinese pre-school children. J Dent Res 2002; 81(11): 767-70.

22. Fung H, Wong MC, Lo EC, Chu C. Arresting early childhood caries with silver diamine fluoride-a literature review. Journal of Oral H ygiene and Health 2013.

23. Dos Santos VE, Jr., Vasconcelos Filho A, Targino AG, Flores MA, Galembeck A, Caldas AF, Jr., et al. A new "silver-bullet" to treat caries in children--nano silver fluoride: a randomised clinical trial. J Dent 2014; 42(8): 945-51.

24. Fatemeh K, Mohammad Javad M, Samaneh K. The effect of silver nanoparticles on composite shear bond strength to dentin with different adhesion protocols. J Appl Oral Sci 2017; 25(4): 367-73.

25. Tirupathi S, Svsg N, Rajasekhar S, Nuvvula S. Comparative cariostatic efficacy of a novel Nano-silver fluoride varnish with $38 \%$ silver diamine fluoride varnish a dou- ble-blind randomized clinical trial. J Clin Exp Dent 2019; 11(2): e105-e12.

26. Faul F, Erdfelder E, Lang A-G, Buchner A. G* Power 3: A flexible statistical power analysis program for the social, behavioral, and biomedical sciences. Behavior research methods 2007; 39(2): 175-91.

27. Wei D, Sun W, Qian W, Ye Y, Ma X. The synthesis of chitosan-based silver nanoparticles and their antibacterial activity. Carbohydr Res 2009; 344(17): 2375-82.

28. Antonson SA, Wanuck J, Antonson DE. Surface protection for newly erupting first molars. Compend Contin Educ Dent 2006; 27(1): 46-52.

29. Malekafzali B, Asnaashari M, Javadi F. Comparison of marginal microleakage of flowable composite restorations in primary canine teeth prepared with high-speed diamond bur, Er:YAG laser and Er,Cr:YSGG laser. Laser Ther 2017; 26(3): 195-202.

30. Garrocho-Rangel A, Lozano-Vázquez C, Butrón-TellezGirón C, Escobar-García D, Ruíz-Rodriguez S, PozosGuillén A. In vitro assessment of retention and microleakage in pit and fissure sealants following enamel pre-etching with sodium hypochlorite deproteinisation. Eur J Paediatr Dent 2015; 16(3): 212-6.

31. Kayapinar U. Measuring essay assessment: Intra-rater and inter-rater reliability. Eurasian J Educ Res 2014; 57: 113-35.

32. Oberholzer TG, Du Preez IC, Kidd M. Effect of LED curing on the microleakage, shear bond strength and surface hardness of a resin-based composite restoration. Biomaterials 2005 ; 26(18): 3981-6.

33. Uzel I, Ulukent O, Cogulu D. The effect of silver diamine fluoride on microleakage of resin composite. J Int Dent Med Res 2013; 6(3): 105.

34. Morales-Quiroga E, Martínez-Sumarán A, Hernández-Sierra JF, Pozos-Guillén A. Evaluation of marginal seal and microleakage of a sealant modified with silver nanoparticles in primary molars: In vitro study. Odovtos Int J Dent Sci 2014; (16): 107-13.

35. Khairy SM, El-Tekeya MM, Bakry NS, Aboshelib MN. Evaluation of Microleakage of Silver Nanoparticle-Added Pit and Fissure Sealant in Permanent Teeth (In-Vitro Study). Alexandria Dental Journal 2020.

36. Suzuki T. Effects of diammine silver fluoride on tooth enamel. J Osaka Univ Dent Sch 1974; 14: 61-72. 